\title{
3D-2D ASYMPTOTIC ANALYSIS FOR MICROMAGNETIC THIN FILMS
}

\author{
Roberto Alicandro ${ }^{1}$ And Chiara Leone ${ }^{2}$
}

\begin{abstract}
Gamma$-convergence techniques and relaxation results of constrained energy functionals are used to identify the limiting energy as the thickness $\varepsilon$ approaches zero of a ferromagnetic thin structure $\Omega_{\varepsilon}=\omega \times(-\varepsilon, \varepsilon), \omega \subset \mathbb{R}^{2}$, whose energy is given by

$$
\mathcal{E}_{\varepsilon}(\bar{m})=\frac{1}{\varepsilon} \int_{\Omega_{\varepsilon}}\left(W(\bar{m}, \nabla \bar{m})+\frac{1}{2} \nabla \bar{u} \cdot \bar{m}\right) \mathrm{d} x
$$

subject to

and to the constraint$$
\operatorname{div}\left(-\nabla \bar{u}+\bar{m}_{\chi_{\Omega_{\varepsilon}}}\right)=0 \quad \text { on } \mathbb{R}^{3}
$$

$$
|\bar{m}|=1 \text { on } \Omega_{\varepsilon},
$$

where $W$ is any continuous function satisfying $p$-growth assumptions with $p>1$. Partial results are also obtained in the case $p=1$, under an additional assumption on $W$.
\end{abstract}

Mathematics Subject Classification. 35E99, 35M10, 49J45, 74K35.

Received September 25, 2000. Revised April, 2001.

\section{INTRODUCTION}

In recent years the understanding of thin film behavior has been helped by the mathematical asymptotic analysis of energies defined on three-dimensional domains of vanishing thickness, through the use of $\Gamma$-convergence techniques (see $[2,3,9,10,12]$ ). The method consists in rescaling the $\varepsilon$-thin domain into a reference body of unit thickness, so that the resulting energy will be defined on a fixed domain, while the dependence on $\varepsilon$ turns out to be explicit in the transversal derivatives which appear in the energy. The second step is then to determine the $\Gamma$-limit of the rescaled energy as the thickness $\varepsilon$ tends to 0.

In this paper and within the framework of micromagnetics, we perform the analysis described above for the energy of a ferromagnetic thin film $\Omega_{\varepsilon}=\omega \times(-\varepsilon, \varepsilon), \omega \subset \mathbb{R}^{2}$, of the type

$$
\mathcal{E}_{\varepsilon}(\bar{m})=\frac{1}{\varepsilon} \int_{\Omega_{\varepsilon}}\left(W(\bar{m}, \nabla \bar{m})+\frac{1}{2} \nabla \bar{u} \cdot \bar{m}\right) \mathrm{d} x
$$

Keywords and phrases: $\Gamma$-limit, thin films, micromagnetics, relaxation of constrained functionals.

1 SISSA, Via Beirut 4, 34013 Trieste, Italy; e-mail: alicandr@sissa.it

2 Dipartimento di Matematica, Università di Roma I, P.le A. Moro 2, 00185 Roma, Italy; e-mail: leone@mat.uniroma1.it 
subject to

$$
\operatorname{div}\left(-\nabla \bar{u}+\bar{m} \chi_{\Omega_{\varepsilon}}\right)=0 \quad \text { on } \mathbb{R}^{3},
$$

and to the constraint

$$
|\bar{m}|=1 \text { on } \Omega_{\varepsilon},
$$

where $W$ is any continuous function satisfying $p$-growth assumptions, with $p \geq 1$ (see Sect. 4 ). Here $\bar{m}: \Omega_{\varepsilon} \rightarrow \mathbb{R}^{3}$ represents the magnetization and $\bar{u}$ is a scalar potential for the magnetic field $H=-\nabla \bar{u}$. Our study generalizes the case studied by Gioia and James in [12] (see Rem. 4.7), where

$$
W(\bar{m}, \nabla \bar{m})=\gamma|\nabla \bar{m}|^{2}+\varphi(\bar{m})
$$

and $\mathcal{E}_{\varepsilon}$ represents the standard micromagnetic energy (see [4] and [12] for a detailed explanation of the model).

In our analysis a fundamental role is played by the characterization of the relaxation of integral functionals where the admissible fields are constrained to remain on the unit sphere. This problem has been faced in [7], where the notion of tangential quasiconvexification $Q_{T} f$ of a function $f$ has been introduced (see Def. 3.1). For $p>1$ we show that the limit energy is

$$
E(m)=\int_{\omega} 2 Q_{T} \hat{W}(m, \nabla m)+m_{3}^{2} \mathrm{~d} x_{1} \mathrm{~d} x_{2} \quad \text { on } W^{1, p}(\omega) \cap\{|m|=1\},
$$

where $\hat{W}$ is obtained by $W$ through a minimization with respect to the transversal derivatives of $m$ (see formula (4.11)). Thus in the limit the admissible fields do not depend on the direction normal to the thin film, and the term $\int_{\omega}\left|m_{3}\right|^{2}$ is the limit of the rescaled version of the magnetostatic energy $\frac{1}{\varepsilon} \int_{\Omega_{\varepsilon}} \frac{1}{2} \nabla \bar{u} \cdot \bar{m}$ (see Prop. 4.1).

So in the superlinear case $(p>1)$ we completely characterize the $\Gamma$-limit of the rescaled version of $(1.1)$ and we derive a convergence result of minimum problems.

In the case $p=1$, the characterization of the $\Gamma$-limit is not completely obtained, since it relies on the still open problem of finding an integral representation of relaxed functionals of the type

$$
\mathcal{F}(u)=\inf \left\{\liminf _{n} \int_{\Omega} f\left(u_{n}, \nabla u_{n}\right) \mathrm{d} x, u_{n} \rightarrow u \text { in } L^{1}(\Omega),\left\|\nabla u_{n}\right\|_{1} \leq c, u_{n} \in W^{1,1}(\Omega),\left|u_{n}\right|=1\right\}
$$

when $f$ is a continuous function with linear growth. In this case, the natural space where to set the problem is $B V\left(\Omega, \mathcal{S}^{2}\right)$, the functions of bounded variation with values on the unit sphere $\mathcal{S}^{2}$. An integral representation of $\mathcal{F}(u)$, depending also on the singular part of $D u$ with respect to the Lebesgue measure, is a problem on which the authors are working. As a partial result, in this paper, we characterize $\mathcal{F}$ on $W^{1,1}\left(\Omega, \mathcal{S}^{2}\right)($ see Th. 3.4). This allows us to show that, in the case $p=1, E(m)$ still has the same expression (1.3) on $W^{1,1}(\omega) \cap\{|m|=1\}$.

The outline of the paper is the following. In Section 2 we recall the definitions and the main properties of relaxation and $\Gamma$-convergence. In Section 3 we state the main result concerning relaxation of constrained integral functionals obtained in [7], and in Theorem 3.4 we prove some extensions of this result to the linear case. Finally, Section 4 is devoted to the characterization of the $\Gamma$-limit of the rescaled version of (1.1) both in the superlinear and in the linear case (see Ths. 4.2 and 4.8). 


\section{Relaxation and T-COnVERGence}

We first recall the notion of relaxed functional. Let $(X, \mathcal{T})$ be a topological space and $F: X \rightarrow[0,+\infty]$. Then the relaxed functional $\bar{F}$ of $F$, or relaxation of $F$, is the greatest $\mathcal{T}$-lower semicontinuous functional less than or equal to $F$. We also give the notion of sequential relaxation of $F$ :

$$
\bar{F}(u)=\inf \left\{\liminf _{j} F\left(u_{j}\right): u_{j} \rightarrow u \text { in } X\right\} .
$$

If $\mathcal{T}$ is induced by a metric, then the two notions are equivalent.

Let $(X, d)$ be a metric space. A family $\left(F_{\varepsilon}\right)_{\varepsilon>0}$ of functionals $F_{\varepsilon}: X \rightarrow[0,+\infty]$ is said to $\Gamma$-converge to a functional $F: X \rightarrow[0,+\infty]$ at $u \in X$, and we write $F(u)=\Gamma-\lim _{\varepsilon \rightarrow 0^{+}} F_{\varepsilon}(u)$, if for every sequence $\left(\varepsilon_{j}\right)$ of positive numbers decreasing to 0 the following two conditions hold:

(i) (lower semicontinuity inequality) for all sequences $\left(u_{j}\right)$ converging to $u$ in $X$ we have $F(u) \leq \liminf _{j} F_{\varepsilon_{j}}\left(u_{j}\right)$;

(ii) (existence of a recovery sequence) there exists a sequence $\left(u_{j}\right)$ converging to $u$ in $X$ such that $F(u) \geq$ $\lim \sup _{j} F_{\varepsilon_{j}}\left(u_{j}\right)$.

We say that $F_{\varepsilon} \Gamma$-converges to $F$ if $F(u)=\Gamma$ - $\lim _{\varepsilon \rightarrow 0^{+}} F_{\varepsilon}(u)$ at all points $u \in X$ and that $F$ is the $\Gamma$-limit of $F_{\varepsilon}$. If we define the lower and upper $\Gamma$-limits by

$$
\begin{aligned}
& F^{\prime \prime}(u)=\Gamma-\limsup _{\varepsilon \rightarrow 0+} F_{\varepsilon}(u)=\inf \left\{\limsup _{\varepsilon \rightarrow 0} F_{\varepsilon}\left(u_{\varepsilon}\right): u_{\varepsilon} \rightarrow u \text { in } X\right\}, \\
& F^{\prime}(u)=\Gamma-\liminf _{\varepsilon \rightarrow 0+} F_{\varepsilon}(u)=\inf \left\{\liminf _{\varepsilon \rightarrow 0} F_{\varepsilon}\left(u_{\varepsilon}\right): u_{\varepsilon} \rightarrow u \text { in } X\right\},
\end{aligned}
$$

respectively, then the conditions (i) and (ii) are equivalent to $F^{\prime}(u)=F^{\prime \prime}(u)=F(u)$. Note that the functions $F^{\prime}$ and $F^{\prime \prime}$ are lower semicontinuous. Moreover if $F_{\varepsilon} \equiv F$, for every $\varepsilon>0$, then $F^{\prime}=F^{\prime \prime}=\bar{F}$, the relaxation of $F$.

A fundamental result concerning the notion of $\Gamma$-convergence is the following theorem:

Theorem 2.1. Let $F=\Gamma-\lim _{\varepsilon \rightarrow 0^{+}} F_{\varepsilon}$, and let $K \subset X$ be a compact set such that $\inf _{X} F_{\varepsilon}=\inf _{K} F_{\varepsilon}$ for all $\varepsilon$. Then $F$ attains its minimum on $X$ and

$$
\min _{X} F=\lim _{\varepsilon \rightarrow 0^{+}} \inf _{X} F_{\varepsilon}
$$

Moreover, if $\left(u_{j}\right)$ is a converging sequence such that $\lim _{j} F_{\varepsilon_{j}}\left(u_{j}\right)=\lim _{j} \inf _{X} F_{\varepsilon_{j}}$ then its limit is a minimum point for $F$.

We refer to [8] for an exposition of the main properties of $\Gamma$-convergence (see also [1]).

\section{RELAXATION OF CONSTRAINED ENERGY FUNCTIONALS}

Let $\Omega$ be a bounded, open set of $\mathbb{R}^{N}$, and let $f: \mathbb{R}^{d} \times \mathbb{R}^{d \times N} \rightarrow[0,+\infty)$ be a continuous function.

The quasiconvex envelope $Q f$ of $f$ is defined by

$$
Q f(y, \xi)=\inf \left\{\int_{(0,1)^{N}} f(y, \xi+\nabla \varphi(x)) \mathrm{d} x: \varphi \in W_{0}^{1, \infty}\left((0,1)^{N}, \mathbb{R}^{d}\right)\right\}
$$

(see [13] and [14]). It has been shown by Dacorogna (see [6]) that, if $f$ satisfies

$$
0 \leq f(y, \xi) \leq C\left(1+|\xi|^{p}\right), \quad \forall(y, \xi) \in \mathbb{R}^{d} \times \mathbb{R}^{d \times N}
$$


for some $C>0, p>1$, then the sequential relaxed energy

$$
\mathcal{F}(u)=\inf \left\{\liminf _{n} \int_{\Omega} f\left(u_{n}, \nabla u_{n}\right) \mathrm{d} x: u_{n} \rightarrow u \text { in } W^{1, p}\left(\Omega, \mathbb{R}^{d}\right)\right\}
$$

is given by

$$
\mathcal{F}(u)=\int_{\Omega} Q f(u, \nabla u) \mathrm{d} x
$$

The integral representation of the relaxed energy when the admissible fields are constrained to remain on a $C^{1}$ manifold $\mathcal{M} \subset \mathbb{R}^{d}$, has been studied in [7].

Let us consider the case $\mathcal{M}=\mathcal{S}^{d-1}$, the unit sphere in $\mathbb{R}^{d}$. If $y \in \mathcal{S}^{d-1}$, we denote by $T_{y}\left(\mathcal{S}^{d-1}\right)$ the tangent space to $\mathcal{S}^{d-1}$ at $y$. Recall that $T_{y}\left(\mathcal{S}^{d-1}\right)=y^{\perp}$, the linear hyperplane orthogonal to $y$.

The following definition was introduced in [7], when $f$ does not depend on $y$.

Definition 3.1. Let $y \in \mathcal{S}^{d-1}$ and $\xi \in\left[T_{y}\left(\mathcal{S}^{d-1}\right)\right]^{N}$. The tangential quasiconvexification of $f$ at $(y, \xi)$ is defined by

$$
Q_{T}^{N, d} f(y, \xi)=\inf \left\{\int_{(0,1)^{N}} f(y, \xi+\nabla \varphi(x)) \mathrm{d} x: \varphi \in W_{0}^{1, \infty}\left((0,1)^{N}, T_{y}\left(\mathcal{S}^{d-1}\right)\right)\right\} .
$$

We say that $f$ is a tangential quasiconvex function if $f(y, \xi)=Q_{T}^{N, d} f(y, \xi)$, for all $y \in \mathcal{S}^{d-1}$ and $\xi \in\left[T_{y}\left(\mathcal{S}^{d-1}\right)\right]^{N}$.

Setting, for any $(y, \xi) \in \mathbb{R}^{d} \times \mathbb{R}^{d \times N}$,

$$
\bar{f}(y, \xi)= \begin{cases}\min \{|y|, 1\} f\left(\frac{y}{|y|},\left(I_{d \times d}-\frac{y \otimes y}{|y|^{2}}\right) \xi\right) & y \neq 0 \\ 0 & y=0,\end{cases}
$$

we can prove, as in Proposition 2.2 of [7], that, for $y \in \mathcal{S}^{d-1}$ and $\xi \in\left[T_{y}\left(\mathcal{S}^{d-1}\right)\right]^{N}$,

$$
Q_{T}^{N, d} f(y, \xi)=Q \bar{f}(y, \xi) .
$$

Let us note that, if $y \in \mathcal{S}^{d-1}$, then $\left(I_{d \times d}-y \otimes y\right) \xi=\left(P_{y} \xi^{1}, \ldots, P_{y} \xi^{N}\right)$, for any $\xi=\left(\xi^{1}, \ldots, \xi^{N}\right) \in \mathbb{R}^{d \times N}$, where $P_{y}$ is the orthogonal projection of $\mathbb{R}^{d}$ onto the tangent space $T_{y}\left(\mathcal{S}^{d-1}\right)$.

For any $u \in W^{1, p}\left(\Omega, \mathcal{S}^{d-1}\right)$ define

$$
\mathcal{F}_{T}(u)=\inf \left\{\liminf _{n} \int_{\Omega} f\left(u_{n}, \nabla u_{n}\right) \mathrm{d} x: u_{n} \rightarrow u \text { in } W^{1, p}\left(\Omega, \mathbb{R}^{d}\right), u_{n} \in \mathcal{S}^{d-1} \text { a.e. in } \Omega\right\} .
$$

The following result is a slight generalization of Theorem 3.1 in [7]. We omit the proof since it does not require any improvement of the argument used in [7].

Theorem 3.2. Let $f: \mathbb{R}^{d} \times \mathbb{R}^{d \times N} \rightarrow[0,+\infty)$ be a continuous function such that

$$
0 \leq f(y, \xi) \leq C\left(1+|\xi|^{p}\right), \quad \forall(y, \xi) \in \mathbb{R}^{d} \times \mathbb{R}^{d \times N},
$$

for some $C>0, p \geq 1$. Then for every $u \in W^{1, p}\left(\Omega, \mathcal{S}^{d-1}\right)$

$$
\mathcal{F}_{T}(u)=\int_{\Omega} Q_{T}^{N, d} f(u, \nabla u) \mathrm{d} x .
$$


Remark 3.3. Let $f$ satisfy (3.5) and the additional coerciveness assumption

$$
C_{1}|\xi|^{p} \leq f(y, \xi), \quad \forall(y, \xi) \in \mathbb{R}^{d} \times \mathbb{R}^{d \times N}
$$

for some constant $C_{1}>0$, and define the functional

$$
F(u)=\left\{\begin{array}{lll}
\int_{\Omega} f(u, \nabla u) \mathrm{d} x & \text { if } & u \in W^{1, p}\left(\Omega, \mathcal{S}^{d-1}\right) \\
+\infty & \text { if } & u \in L^{1}\left(\Omega, \mathbb{R}^{d}\right) \backslash W^{1, p}\left(\Omega, \mathcal{S}^{d-1}\right) .
\end{array}\right.
$$

Then, for $p>1$, as a straightforward consequence of the previous result, we have that the relaxation of $F$ with respect to the $L^{1}$-metric

$$
\bar{F}(u)=\inf \left\{\liminf _{n} F\left(u_{n}\right): u_{n} \rightarrow u \text { in } L^{1}\left(\Omega, \mathbb{R}^{d}\right)\right\}
$$

has the following integral representation

$$
\bar{F}(u)=\left\{\begin{array}{lll}
\int_{\Omega} Q_{T}^{N, d} f(u, \nabla u) \mathrm{d} x & \text { if } & u \in W^{1, p}\left(\Omega, \mathcal{S}^{d-1}\right) \\
+\infty & \text { if } & u \in L^{1}\left(\Omega, \mathbb{R}^{d}\right) \backslash W^{1, p}\left(\Omega, \mathcal{S}^{d-1}\right) .
\end{array}\right.
$$

If $p=1$, as a partial result, the following theorem characterizes $\bar{F}$ on $W^{1,1}\left(\Omega, \mathcal{S}^{d-1}\right)$ and it is based on the lower semicontinuity results of [11].

Theorem 3.4. Let $f$ be a continuous function satisfying (3.5) and (3.6), with $p=1$, and let $\bar{f}$, given in (3.3), satisfy the following hypothesis

(i) for all $y_{0} \in \mathbb{R}^{d}$ and for all $\eta>0$ there exists $\delta>0$ such that $\left|y-y_{0}\right|<\delta$ implies that

$$
\bar{f}\left(y_{0}, \xi\right)-\bar{f}(y, \xi) \leq \eta(1+|\xi|) .
$$

Then

$$
\bar{F}(u)=\int_{\Omega} Q_{T}^{N, d} f(u, \nabla u) \mathrm{d} x \quad \text { on } W^{1,1}\left(\Omega, \mathcal{S}^{d-1}\right) .
$$

Proof. From Theorem 3.2 it follows that

$$
\bar{F}(u) \leq \int_{\Omega} Q_{T}^{N, d} f(u, \nabla u) \mathrm{d} x \quad \text { on } W^{1,1}\left(\Omega, \mathcal{S}^{d-1}\right) .
$$

The opposite inequality is a consequence of (3.4). Indeed, if $u \in W^{1,1}\left(\Omega, \mathcal{S}^{d-1}\right)$ we have that $\nabla u \in\left[T_{u}\left(\mathcal{S}^{d-1}\right)\right]^{N}$ a.e. in $\Omega$, thus

$$
f(u, \nabla u)=\bar{f}(u, \nabla u) \quad \text { a.e. in } \Omega
$$

which implies

$$
F(u)=\int_{\Omega} \bar{f}(u, \nabla u) \mathrm{d} x \quad \text { on } W^{1,1}\left(\Omega, \mathcal{S}^{d-1}\right)
$$

Then, by virtue of (3.4) and the coerciveness assumption (3.6), we get the conclusion if we prove that for any $u_{n}, u \in W^{1,1}\left(\Omega, \mathbb{R}^{d}\right)$, with $u_{n}$ converging to $u$ strongly in $L^{1}\left(\Omega, \mathbb{R}^{d}\right)$ and $u_{n}$ bounded in $W^{1,1}\left(\Omega, \mathbb{R}^{d}\right)$,

$$
\liminf _{n} \int_{\Omega} \bar{f}\left(u_{n}, \nabla u_{n}\right) \mathrm{d} x \geq \int_{\Omega} Q \bar{f}(u, \nabla u) \mathrm{d} x
$$


This can be done following the line of the proof of Theorem 2.1 in [11], up to slight modifications. Note that the hypothesis (i) corresponds to condition (H4) in [11] and (3.5) yields

$$
0 \leq \bar{f}(y, \xi) \leq C(1+|\xi|), \quad(y, \xi) \in \mathbb{R}^{d} \times \mathbb{R}^{d \times N} .
$$

The hypothesis of coerciveness on $\bar{f}$, required in Theorem 2.1 of [11], is not needed by the boundedness of $u_{n}$ in $W^{1,1}\left(\Omega, \mathbb{R}^{d}\right)$.

Remark 3.5. Note that, even if the function $f$ does not satisfy the coerciveness condition (3.6), the same conclusion of Theorem 3.4 still holds for the functional

$$
\tilde{F}(u)=\inf \left\{\liminf _{n} F\left(u_{n}\right): u_{n} \rightarrow u \text { in } L^{1}\left(\Omega, \mathbb{R}^{d}\right),\left(u_{n}\right) \text { bounded in } W^{1,1}\left(\Omega, \mathbb{R}^{d}\right)\right\} .
$$

Remark 3.6. It is easy to prove that, if $f(y, \xi)$ is Lipschitz in $\xi$ uniformly with respect to $y$, the hypothesis (i) on $\bar{f}$ in Theorem 3.4 is implied by the same hypothesis on $f$.

\section{Limit OF MICROMAGNETIC ENERGIES ON THIN FILMS}

For $\varepsilon>0$ let $\Omega_{\varepsilon}$ be a thin three-dimensional domain of the form $\Omega_{\varepsilon}=\omega \times(-\varepsilon, \varepsilon)$, with $\omega$ a bounded open set of $\mathbb{R}^{2}$, and denote $\Omega:=\Omega_{1}$. Let $p \geq 1$ and let $W: \mathbb{R}^{3} \times \mathbb{R}^{3 \times 3} \rightarrow \mathbb{R}$ be a continuous function such that, for any $(y, \xi) \in \mathbb{R}^{3} \times \mathbb{R}^{3 \times 3}$,

$$
\frac{1}{C}|\xi|^{p} \leq W(y, \xi) \leq C\left(1+|\xi|^{p}\right)
$$

for some constant $C>0$.

Define, for any $\bar{m} \in L^{1}\left(\Omega_{\varepsilon}, \mathbb{R}^{3}\right)$,

$$
\mathcal{E}_{\varepsilon}(\bar{m})= \begin{cases}\frac{1}{\varepsilon} \int_{\Omega_{\varepsilon}}\left(W(\bar{m}, \nabla \bar{m})+\frac{1}{2} \nabla \bar{u} \cdot \bar{m}\right) \mathrm{d} x & \text { if } \bar{m} \in W^{1, p}\left(\Omega_{\varepsilon}, \mathcal{S}^{2}\right) \\ +\infty & \text { otherwise }\end{cases}
$$

where $\bar{u}$ is related to $\bar{m}$ by the equation

$$
\operatorname{div}(-\nabla \bar{u}+\bar{m})=0 \quad \text { on } \mathbb{R}^{3}
$$

with $\bar{m}$ extended by 0 outside $\Omega_{\varepsilon}$.

Through the change of variables

$$
m\left(x_{\alpha}, x_{3}\right)=\bar{m}\left(x_{\alpha}, \varepsilon x_{3}\right), \quad u\left(x_{\alpha}, x_{3}\right)=\bar{u}\left(x_{\alpha}, \varepsilon x_{3}\right), \quad x_{\alpha}=\left(x_{1}, x_{2}\right) \in \omega, x_{3} \in(-1,1),
$$

we rescale the functional (4.2) as

$$
E_{\varepsilon}(m)= \begin{cases}\int_{\Omega} W\left(m, \nabla_{\alpha} m, \frac{1}{\varepsilon} \nabla_{3} m\right) \mathrm{d} x+\frac{1}{2} \int_{\mathbb{R}^{3}}\left(\nabla_{\alpha} u \cdot m_{\alpha}+\frac{1}{\varepsilon} m_{3} \nabla_{3} u\right) \mathrm{d} x & \text { if } m \in W^{1, p}\left(\Omega, \mathcal{S}^{2}\right) \\ +\infty & \text { otherwise }\end{cases}
$$

subjected to the constraint

$$
\operatorname{div}\left(-\nabla_{\alpha} u+m_{\alpha}\right)+\frac{1}{\varepsilon} \nabla_{3}\left(-\frac{1}{\varepsilon} \nabla_{3} u+m_{3}\right)=0 \quad \text { on } \mathbb{R}^{3}
$$


where we have used the notation

$$
m_{\alpha}=\left(m_{1}, m_{2}\right), \quad \nabla_{i}=\frac{\partial}{\partial x_{i}}, i=1,2,3, \quad \nabla_{\alpha}=\left(\nabla_{1}, \nabla_{2}\right)
$$

We now proceed to clarify the meaning of the magnetostatic equation (4.6). Consider the following variational principle

$$
\min _{u \in V} \frac{1}{2} \int_{\mathbb{R}^{3}}\left|\nabla_{\alpha} u-m_{\alpha}\right|^{2}+\left|\frac{1}{\varepsilon} \nabla_{3} u-m_{3}\right|^{2} \mathrm{~d} x
$$

where

$$
V=\left\{v \in L_{\text {loc }}^{1}\left(\mathbb{R}^{3}\right): \nabla v \in L^{2}\left(\mathbb{R}^{3}, \mathbb{R}^{3}\right), \int_{B} v \mathrm{~d} x=0\right\},
$$

and $B$ is a fixed ball of $\mathbb{R}^{N}$. $V$ is a Hilbert space with inner product

$$
(u, v)_{V}=\int_{\mathbb{R}^{3}}\left(\nabla_{\alpha} u \cdot \nabla_{\alpha} v+\frac{1}{\varepsilon^{2}} \nabla_{3} u \nabla_{3} v\right) \mathrm{d} x .
$$

The direct method of the calculus of variations yields a unique minimizer of (4.7) in $V$, satisfying the Euler-Lagrange equation

$$
\int_{\mathbb{R}^{3}}\left(\nabla_{\alpha} u-m_{\alpha}\right) \cdot \nabla_{\alpha} v+\frac{1}{\varepsilon}\left(\frac{1}{\varepsilon} \nabla_{3} u-m_{3}\right) \nabla_{3} v \mathrm{~d} x=0 \quad \forall v \in V,
$$

that is the weak form of (4.6). Setting $v=u$ in (4.8), and taking into account that $m$ vanishes outside $\Omega$, we obtain

$$
\int_{\Omega} \nabla_{\alpha} u \cdot m_{\alpha}+\frac{1}{\varepsilon} m_{3} \nabla_{3} u \mathrm{~d} x=\int_{\mathbb{R}^{3}}\left|\nabla_{\alpha} u\right|^{2}+\frac{1}{\varepsilon^{2}}\left|\nabla_{3} u\right|^{2} \mathrm{~d} x .
$$

Note that the left-hand side of this expression is twice the magnetostatic energy given by the second integral in (4.5).

The following proposition is due to Gioia and James (Prop. 4.1 of [12]).

Proposition 4.1. Suppose $m_{\varepsilon} \rightarrow m$ in $L^{2}\left(\mathbb{R}^{3}, \mathbb{R}^{3}\right), m_{\varepsilon}=0$ on $\mathbb{R}^{3} \backslash \Omega$, and let $u_{\varepsilon}$ be the minimizer of (4.7) corresponding to $m_{\varepsilon}$. Then

$$
\nabla u_{\varepsilon} \rightarrow 0, \quad \frac{1}{\varepsilon} \nabla_{3} u_{\varepsilon} \rightarrow m_{3} \quad \text { in } L^{2}\left(\mathbb{R}^{3}\right)
$$

We now state the thin-film approximation result in the superlinear case.

Theorem 4.2. If $p>1$, then $E_{\varepsilon} \Gamma$-converges with respect to the $L^{1}$-strong topology to the functional $E$ : $\mathrm{L}^{1}\left(\Omega, \mathbb{R}^{3}\right) \rightarrow[0,+\infty]$ defined as

$$
E(m)= \begin{cases}2 \int_{\omega} Q_{T}^{2,3} \hat{W}(m, \nabla m) \mathrm{d} x_{\alpha}+\int_{\omega}\left|m_{3}\right|^{2} \mathrm{~d} x_{\alpha} & \text { if } m \in W^{1, p}\left(\omega, \mathcal{S}^{2}\right) \\ +\infty & \text { otherwise }\end{cases}
$$

where $\hat{W}: \mathbb{R}^{3} \times \mathbb{R}^{3 \times 2} \rightarrow \mathbb{R}$ is given by

$$
\hat{W}(y, \zeta):=\inf _{z \in y^{\perp}} W(y, \zeta, z)
$$


Remark 4.3. It is easy to check, by the continuity and the growth assumptions (4.1) on $W$, that $\hat{W}$ satisfies the hypotheses of Theorem 3.2 .

Proof of Theorem 4.2. As usual we divide the proof in two steps dealing with the $\Gamma$-liminf and $\Gamma$-limsup inequalities, separately.

Step 1. $\Gamma-\liminf \operatorname{s\rightarrow 0}_{\varepsilon \rightarrow 0} E_{\varepsilon}(m) \geq E(m)$ for any $m \in \mathrm{L}^{1}\left(\Omega, \mathbb{R}^{3}\right)$.

Let $\varepsilon_{h} \searrow 0$ and let $m_{h} \rightarrow m$ in $\mathrm{L}^{1}\left(\Omega, \mathbb{R}^{3}\right)$ be such that $\liminf _{h} E_{\varepsilon_{h}}\left(m_{h}\right)<+\infty$. Then, up to a subsequence, by the coerciveness assumption (4.1), we have that $m_{h} \in W^{1, p}\left(\Omega, \mathcal{S}^{2}\right)$ and

$$
\sup _{h} \int_{\Omega}\left|\nabla_{\alpha} m_{h}\right|^{p}+\frac{1}{\varepsilon_{h}^{p}}\left|\nabla_{3} m_{h}\right|^{p} \mathrm{~d} x<+\infty .
$$

Thus $m_{h}$ converges to $m$ weakly in $W^{1, p}\left(\Omega, \mathbb{R}^{3}\right)$ and strongly in $L^{q}\left(\Omega, \mathbb{R}^{3}\right)$, for every $q<+\infty$, since $\left\|m_{h}\right\|_{L^{\infty}(\Omega)}$ is equi-bounded. Moreover, $m \in W^{1, p}\left(\Omega, \mathcal{S}^{2}\right)$, and $\nabla_{3} m=0$, that is $m$ does not depend on the transverse direction $x_{3}$.

Since $\nabla_{3} m_{h} \in m_{h}^{\perp}$ and (4.9) holds, we have

$$
E_{\varepsilon_{h}}\left(m_{h}\right) \geq \int_{\Omega} \hat{W}\left(m_{h}, \nabla_{\alpha} m_{h}\right) \mathrm{d} x+\frac{1}{2} \int_{\Omega}\left|\nabla_{\alpha} u_{h}\right|^{2}+\frac{1}{\varepsilon_{h}^{2}}\left|\nabla_{3} u_{h}\right|^{2} \mathrm{~d} x,
$$

where $u_{h}$ is the solution of (4.6) corresponding to $m_{h}$. Then Theorem 3.2 and Proposition 4.1 yield

$$
\underset{h}{\liminf } E_{\varepsilon_{h}}\left(m_{h}\right) \geq \int_{\Omega} Q_{T}^{3,3} \hat{W}\left(m, \nabla_{\alpha} m\right) \mathrm{d} x+\frac{1}{2} \int_{\Omega}\left|m_{3}\right|^{2} \mathrm{~d} x .
$$

A straightforward application of Fubini's theorem in the definition (3.2) shows that $Q_{T}^{3,3} \hat{W}(m, \zeta) \geq Q_{T}^{2,3} \hat{W}(m, \zeta)$, $(m, \zeta) \in \mathbb{R}^{3} \times \mathbb{R}^{3 \times 2}$. By the arbitrariness of the sequence $\left(\varepsilon_{h}\right)$, we get the conclusion.

Step 2. $\Gamma$ - $\lim \sup _{\varepsilon \rightarrow 0} E_{\varepsilon}(m) \leq E(m)$, for any $m \in \mathrm{L}^{1}\left(\Omega, \mathbb{R}^{3}\right)$.

By the lower semicontinuity of $\Gamma$-limsup and by Theorem 3.2 and Remark 3.3, it suffices to show that, for every $m \in W^{1, p}\left(\omega, \mathcal{S}^{2}\right)$,

$$
\Gamma-\limsup _{\varepsilon \rightarrow 0} E_{\varepsilon}(m) \leq 2 \int_{\omega} \hat{W}(m, \nabla m) \mathrm{d} x_{\alpha}+\int_{\omega}\left|m_{3}\right|^{2} \mathrm{~d} x_{\alpha} .
$$

Let $m \in W^{1, p}\left(\omega, \mathcal{S}^{2}\right)$. For any $\eta>0$, a measurability selection criterion (see [5] for example) allows us to find a measurable function $\tilde{z}: \omega \rightarrow \mathbb{R}^{3}$ such that $\tilde{z}(x) \in m(x)^{\perp}$ a.e. in $\omega$ and

$$
\int_{\omega} \hat{W}(m, \nabla m) \mathrm{d} x_{\alpha} \geq \int_{\omega} W(m, \nabla m, \tilde{z}) \mathrm{d} x_{\alpha}-\eta .
$$

The growth assumptions on $W$ and $\hat{W}$ ensure that $\tilde{z} \in L^{p}\left(\omega, \mathbb{R}^{3}\right)$. Consider a sequence $\tilde{z}_{n} \in C_{c}^{\infty}\left(\omega, \mathbb{R}^{3}\right)$ which approximates $\tilde{z}$ strongly in $L^{p}\left(\omega, \mathbb{R}^{3}\right)$, and set

$$
z_{n}=(I-m \otimes m) \tilde{z}_{n},
$$

that is the projection of $\tilde{z}_{n}$ on $m^{\perp}$. We have that $z_{n} \in W^{1, p}\left(\omega, \mathbb{R}^{3}\right) \cap L^{\infty}\left(\omega, \mathbb{R}^{3}\right)$ and $z_{n}$ still converges to $\tilde{z}$ strongly in $L^{p}\left(\omega, \mathbb{R}^{3}\right)$. Then, we can find $n(\eta)$ such that

$$
\int_{\omega} \hat{W}(m, \nabla m) \mathrm{d} x_{\alpha} \geq \int_{\omega} W\left(m, \nabla m, z_{n(\eta)}\right) \mathrm{d} x_{\alpha}-2 \eta .
$$


Set $z=z_{n(\eta)}$ and define, for $\varepsilon>0$,

$$
\bar{m}_{\varepsilon}(x)=m\left(x_{\alpha}\right)+\varepsilon x_{3} z\left(x_{\alpha}\right), \quad m_{\varepsilon}(x)=\frac{\bar{m}_{\varepsilon}(x)}{\left|\bar{m}_{\varepsilon}(x)\right|}, \quad x \in \Omega .
$$

Since $z \in W^{1, p}\left(\omega, \mathbb{R}^{3}\right) \cap L^{\infty}\left(\omega, \mathbb{R}^{3}\right)$, then, for $\varepsilon$ sufficiently small, $m_{\varepsilon}$ is well defined and belongs to $W^{1, p}\left(\Omega, \mathcal{S}^{2}\right)$; moreover, both $\bar{m}_{\varepsilon}$ and $m_{\varepsilon}$ converge to $m$ strongly in $L^{\infty}\left(\Omega, \mathbb{R}^{3}\right)$. An easy computation shows that

$$
\nabla m_{\varepsilon}=\left(\frac{I}{\left|\bar{m}_{\varepsilon}\right|}-\frac{\bar{m}_{\varepsilon} \otimes \bar{m}_{\varepsilon}}{\left|\bar{m}_{\varepsilon}\right|^{3}}\right) \nabla \bar{m}_{\varepsilon}
$$

In particular, as $\nabla \bar{m}_{\varepsilon}=\left(\nabla m+\varepsilon x_{3} \nabla_{\alpha} z, \varepsilon z\right)$, we have

$$
\nabla_{\alpha} m_{\varepsilon}=\left(\frac{I}{\left|\bar{m}_{\varepsilon}\right|}-\frac{\bar{m}_{\varepsilon} \otimes \bar{m}_{\varepsilon}}{\left|\bar{m}_{\varepsilon}\right|^{3}}\right)\left(\nabla m+\varepsilon x_{3} \nabla_{\alpha} z\right), \quad \nabla_{3} m_{\varepsilon}=\left(\frac{I}{\left|\bar{m}_{\varepsilon}\right|}-\frac{\bar{m}_{\varepsilon} \otimes \bar{m}_{\varepsilon}}{\left|\bar{m}_{\varepsilon}\right|^{3}}\right) \varepsilon z
$$

and, letting $\varepsilon$ tend to 0 ,

$$
\nabla_{\alpha} m_{\varepsilon} \rightarrow(I-m \otimes m) \nabla m=\nabla m, \quad \frac{1}{\varepsilon} \nabla_{3} m_{\varepsilon} \rightarrow(I-m \otimes m) z=z
$$

strongly in $L^{p}\left(\Omega, \mathbb{R}^{3}\right)$.

Thus, by (4.1) and by Proposition 4.1, we get

$$
\lim _{\varepsilon \rightarrow 0} E_{\varepsilon}\left(m_{\varepsilon}\right)=2 \int_{\omega} W(m, \nabla m, z) \mathrm{d} x+\int_{\omega}\left|m_{3}\right|^{2} \mathrm{~d} x \leq 2 \int_{\omega} \hat{W}(m, \nabla m) \mathrm{d} x+\int_{\omega}\left|m_{3}\right|^{2} \mathrm{~d} x+2 \eta .
$$

The inequality (4.12) easily follows by the definition of $\Gamma$-lim sup and the arbitrariness of $\eta>0$.

Remark 4.4. The previous result can be extended to the case of an applied field $h$, by considering functionals of the type

$$
\mathcal{G}_{\varepsilon}(\bar{m})=\mathcal{E}_{\varepsilon}(\bar{m})-\frac{1}{\varepsilon} \int_{\Omega_{\varepsilon}} h \cdot \bar{m} \mathrm{~d} x .
$$

Through the change of variables (4.4), we obtain the functionals

$$
G_{\varepsilon}(m)=E_{\varepsilon}(m)-\int_{\Omega} h\left(x_{\alpha}, \varepsilon x_{3}\right) \cdot m(x) \mathrm{d} x .
$$

In order to identify the $\Gamma$-limit of $G_{\varepsilon}$, a continuity assumption of $h$ in $x_{3}$ is required. By simplicity, we may consider $h(x)=q\left(x_{3}\right) g\left(x_{\alpha}\right)$, with $g \in L^{1}\left(\omega ; \mathbb{R}^{3}\right)$ and $q \in C(-1,1)$. Then it is easy to show that $G_{\varepsilon} \Gamma$-converges to

$$
G(m)=E(m)-2 q(0) \int_{\omega} g \cdot m \mathrm{~d} x_{\alpha} .
$$

Remark 4.5. If $W$ is also a tangential quasiconvex function, the direct method of the calculus of variations yields the existence of a minimizer of $G_{\varepsilon}(m)$.

As a straightforward consequence of Theorem 2.1, we deduce the following result on the convergence of minimum problems. 
Corollary 4.6. Let $W$ be a tangential quasiconvex function, $h$ be as in Remark 4.4, and let $G_{\varepsilon}$ and $G$ be defined by (4.13) and (4.14). If $m_{\varepsilon} \in W^{1, p}\left(\Omega, \mathcal{S}^{2}\right)$ is a minimizer of $G_{\varepsilon}$, then, for every sequence $\left(\varepsilon_{h}\right)$ tending to 0 there exists a subsequence (not relabelled) $\left(m_{\varepsilon_{h}}\right)$ converging weakly in $W^{1, p}\left(\Omega, \mathbb{R}^{3}\right)$ to a function $m \in W^{1, p}\left(\omega, \mathcal{S}^{2}\right)$ which is a minimizer of $G$.

Remark 4.7. If $W(y, \xi)=\gamma|\xi|^{2}+\varphi(y)$, that is $E_{\varepsilon}$ represents the standard micromagnetic energy, we have that

$$
Q_{T}^{2,3} \hat{W}(y, \zeta)=\gamma|\zeta|^{2}+\varphi(y)
$$

and we generalize the convergence result of minimum problems obtained in [12].

If $p=1$, as a consequence of Theorem 3.4 we obtain the following $\Gamma$-convergence result.

Theorem 4.8. Let $p=1$ and let $W$ satisfy the additional hypotheses:

(a) $W(y, \xi)$ is Lipschitz in $\xi$ uniformly with respect to $y$;

(b) for all $y_{0} \in \mathbb{R}^{d}$ and for all $\eta>0$ there exists $\delta>0$ such that $\left|y-y_{0}\right|<\delta$ implies that

$$
W\left(y_{0}, \xi\right)-W(y, \xi) \leq \eta(1+|\xi|)
$$

Then

$$
\Gamma\left(L^{1}\right)-\lim _{\varepsilon \rightarrow 0} E_{\varepsilon}(m)=2 \int_{\omega} Q_{T}^{2,3} \hat{W}(m, \nabla m) \mathrm{d} x_{\alpha}+\int_{\omega}\left|m_{3}\right|^{2} \mathrm{~d} x_{\alpha} \quad \text { on } W^{1,1}\left(\omega, \mathcal{S}^{2}\right) .
$$

Proof. It can be easily proved that hypotheses (a) and (b) are inherited by $\hat{W}$. Then we can proceed as in the proof of Theorem 4.2, by taking into account Theorem 3.4 and Remarks 3.5, 3.6.

Our attention on this problem was drawn by Irene Fonseca. We thank her for many useful discussions concerning the subject of this paper. This research was done while we were postdoctoral associates at the Center for Nonlinear Analysis (NSF Grant No. DMS-9803791) at Carnegie Mellon University of Pittsburgh.

\section{REFERENCES}

[1] A. Braides and A. Defranceschi, Homogenization of Multiple Integrals. Oxford University Press, Oxford (1998).

[2] A. Braides and I. Fonseca, Brittle thin films, Preprint CNA-CMU. Pittsburgh (1999).

[3] A. Braides, I. Fonseca and G. Francfort, 3D-2D asymptotic analysis for inhomogeneous thin films, Preprint CNA-CMU. Pittsburgh (1999).

[4] W.F. Brown, Micromagnetics. John Wiley and Sons, New York (1963).

[5] C. Castaing and M. Valadier, Convex analysis and measurable multifunctions. Springer-Verlag, New York, Lecture Notes in Math. $\mathbf{5 8 0}$ (1977).

[6] B. Dacorogna, Direct methods in Calculus of Variations. Springer-Verlag, Berlin (1989).

[7] B. Dacorogna, I. Fonseca, J. Malỳ and K. Trivisa, Manifold constrained variational problems. Calc. Var. 9 (1999) $185-206$.

[8] G. Dal Maso, An Introduction to $\Gamma$-convergence. Birkhäuser, Boston (1993).

[9] I. Fonseca and G. Francfort, 3D-2D asymptotic analysis of an optimal design problem for thin films. J. Reine Angew. Math. 505 (1998) 173-202.

[10] I. Fonseca and G. Francfort, On the inadequacy of the scaling of linear elasticity for 3D-2D asymptotic in a nonlinear setting, Preprint CNA-CMU. Pittsburgh (1999).

[11] I. Fonseca and S. Müller, Quasi-convex integrands and lower semicontinuity in L ${ }^{1}$. SIAM J. Math. Anal. 23 (1992) $1081-1098$.

[12] G. Gioia and R.D. James, Micromagnetics of very thin films. Proc. Roy. Soc. Lond. Ser. A 453 (1997) 213-223.

[13] C.B. Morrey, Quasiconvexity and the semicontinuity of multiple integrals. Pacific J. Math. 2 (1952) 25-53.

[14] C.B. Morrey, Multiple integrals in the Calculus of Variations. Springer-Verlag, Berlin (1966). 